\title{
Congenital heart defect and vena cava enlargement
}

\author{
Defecto cardíaco congénito y agrandamiento de la vena cava \\ Bárbara Segura*, Gregorio Laguna, Miriam Blanco, and Yolanda Carrascal \\ Department of Cardiac Surgery, Clinic University Hospital of Valladolid, Valladolid, Spain
}

Atrial septal defect (ASD) is the second most frequent congenital heart disease. Only $5-10 \%$ of them are sinus venosus-type ASD (SVASD) and often encompasses a partial anomalous pulmonary venous return (PAPVR).

A 62-year-old man presented a precordial systolic murmur and dyspnea 2 months ago, with right bundle branch block. Transthoracic echocardiogram showed dilated right cavities and severe tricuspid regurgitation. Computerized tomography exposed an extensive SVASD (Fig. 1A, asterisk) associated with a PAPVR $(\mathrm{Qp} / \mathrm{Qs}=3,5)$. The middle (Fig. $1 A$, white arrow) and upper right pulmonary veins (Fig. 1B, white arrow) drained into the superior vena cava (SVC) (Fig. 1B, black arrow). Hemodynamic study showed coronary disease.

PAPVR (Fig. 1C, black arrow) was rerouted and SVASD (Fig. 1C, white arrow) was closed with the same single dented bovine pericardium patch ${ }^{1}$ (Fig. 1D, white arrow), under cardiopulmonary bypass. Subsequently, severe reduction of SVC was found, forcing to enlarge it with an autologous pericardium patch (Fig. 1E, asterisk). Coronary artery bypass grafting (Fig. 1E, white arrow) and rigid tricuspid annuloplasty were undertaken. Post-operative course was uneventful.

PAPVR in adults is an incidental finding and symptomatic patients or associate large SVASDs have indication to surgical correction ${ }^{1,2}$. A rare but serious complication is the reduction of SVC caliber ${ }^{3}$. The SVC narrowing is diagnosed when the patch is sewn, and it is observed because the central venous pressure is higher than in normal conditions. In this case, we needed a large dome-shaped patch to rerouted the venous drainage and close the septal defect with the same patch. This large patch took up space at the cavoatrial junction, reducing the SVC caliber.

There is no standardized surgical technique as a protocol in the treatment of ASD with superior anomalous venous drainage. The peculiarity of this case is that you use the ASD itself to recanalize the anomalous venous drainage and closure the ASD at the same time with a unique patch.

It is important to be aware the appearance of this unexpected event because it could be fixed immediately, to avoid future symptoms (swelling, dyspnea, and coughing) and a SVC syndrome. An advantage of intraoperative diagnosis is the possibility of correction in the same intervention, without the need for a new surgery, reducing the associated morbidity and mortality.

\section{Financial support}

This research has not received any specific scholarships from public, commercial, or non-profit agencies.

\section{Conflicts of interest}

The authors declare that they have had no conflicts of interest.

\section{Correspondence:}

*Barbara Segura

E-mail: barbaraseg@ hotmail.com
Date of reception: 20-11-2019

Date of acceptance: 22-03-2020

DOI: 10.24875/ACM.20000379
Available online: 14-09-2020

Arch Cardiol Mex (Eng). 2020;90(3):347-348 www.archivoscardiologia.com license (http://creativecommons.org/licenses/by-nc-nd/4.0/). 


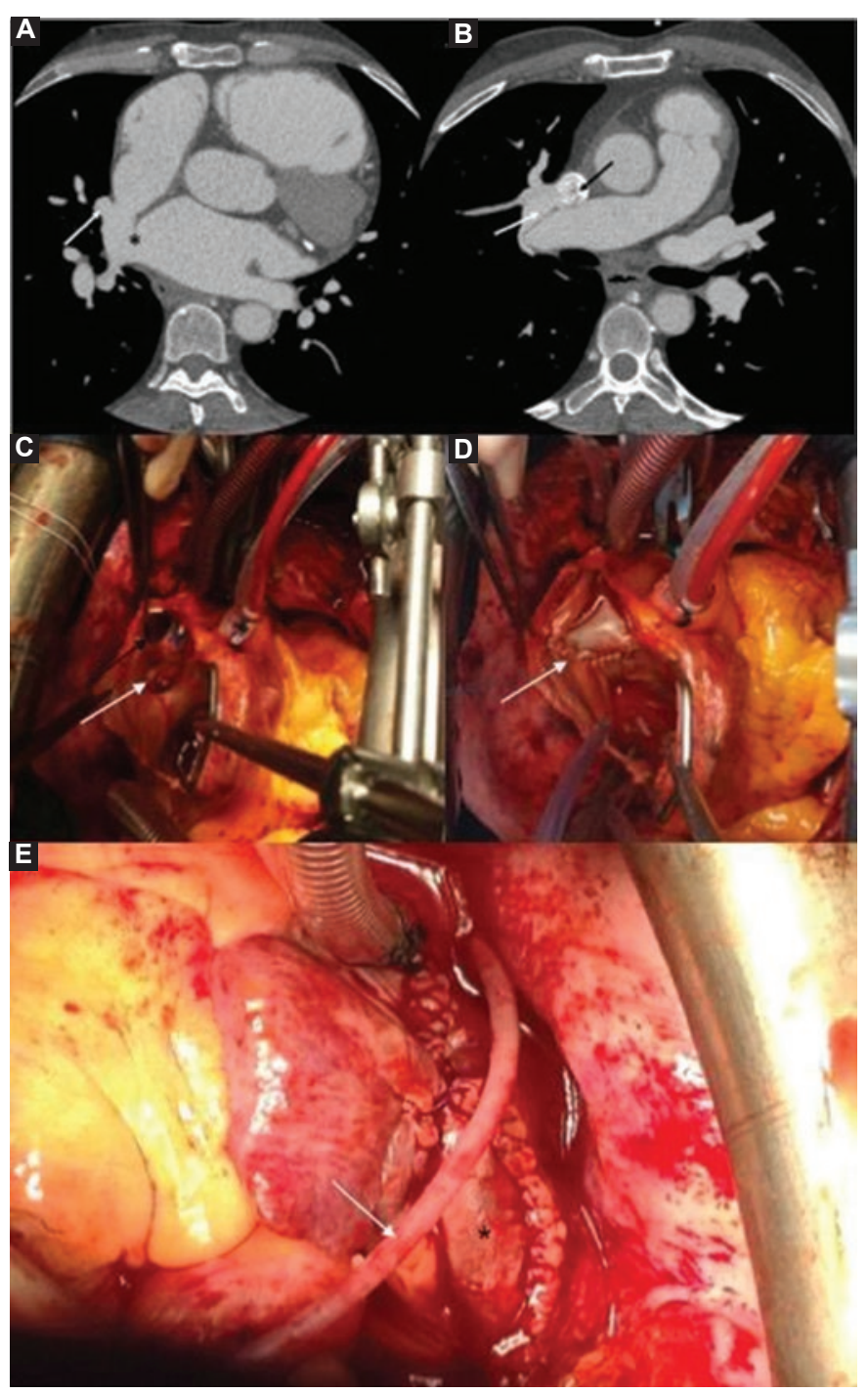

Figure 1. A: Cardiac computerized tomography exposed an extensive sinus venosus-type atrial septal defect (SVASD) (asterisk) associated with a partial anomalous pulmonary venous return (PAPVR). The middle right pulmonary veins drained into the superior vena cava (SVC) (white arrow). B: The upper right pulmonary vein drained into the SVC (black arrow). C: Left atriotomy showed PAPVR (black arrow) and SVASD (white arrow). D: PAPVR was recirculated towards the left atrium and SVASD was closed with the same single dented bovine pericardium patch (white arrow). E: Enlargement of SVC with an autologous pericardium patch (asterisk). In the same procedure, coronary artery bypass grafting (white arrow) was made.

\section{Ethical disclosures}

Protection of human and animal subjects. The authors declare that no experiments were performed on humans or animals for this study.

Confidentiality of data. The authors declare that they have followed the protocols of their work center on the publication of patient data.

Right to privacy and informed consent. The authors have obtained the written informed consent of the patients or subjects mentioned in the article.
The corresponding author is in possession of this document.

\section{References}

1. Iyer AP, Somanrema K, Pathak S, Manjunath PY, Pradhan S, Krishnan S Comparative study of single and double-patch techniques for sinus venosus atrial septal defect with partial anomalous pulmonary venous connection. J Thorac Cardiovasc Surg. 2007:133:656-9.

2. Paulista MD, Paulista PH, Guerra AL, Paulista PP. Surgical treatment of partial anomalous pulmonary venous connection to the superior vena cava. Rev Bras Cir Cardiovasc. 2009;24:133-7.

3. Okonta KE, Tamatey M. Is double or single patch for sinus venosus atrial septal defect repair the better option in prevention of postoperative venous obstruction? Interact Cardiovasc Thorac Surg. 2012;15:900-3. 